\title{
On the Mechanism of the Triplet-to-Singlet Upconversion in the Assistant Dopant ACRXTN Supporting Information
}

\author{
Christel M. Marian* \\ Institute of Theoretical and Computational Chemistry, Heinrich Heine University \\ Düsseldorf, Universitätsstr. 1, D-40225 Düsseldorf, Germany \\ E-mail: Christel.Marian@hhu.de
}

This supplement contains detailed information on selected geometry parameters of all minima optimized in this work and on the vertical excitation energies and spectral properties of the low-lying electronically excited states at these geometries. Further, spin-orbit coupling matrix elements at the singlet $\mathrm{CT}, \mathrm{S}\left(n_{\mathrm{O}} \pi_{L}^{*}\right)$, and $\mathrm{T}\left(\pi_{H-1} \pi_{L}^{*}\right)$ equilibrium geometries are listed.

*To whom correspondence should be addressed 
Table S1: Selected ground and excited-state bond lengths [pm] of ACRXTN. Atomic labels are displayed in Fig. 1 of the main article.

\begin{tabular}{|c|c|c|c|c|c|c|c|c|}
\hline & $\mathrm{GS}^{a}$ & singlet CT & triplet $\mathrm{CT}$ & $\mathrm{S}\left(n_{\mathrm{O}} \pi^{*}\right)$ & $\overline{\mathrm{T}}\left(n_{\mathrm{O}} \pi^{*}\right)$ & $\overline{\mathrm{S}}\left(\pi_{H-1} \pi_{L}^{*}\right)$ & $\overline{\mathrm{T}}\left(\pi_{H-1} \pi_{L}^{*}\right)$ & $\mathrm{GS}^{b}$ \\
\hline$r\left(\mathrm{C}_{1}-\mathrm{C}_{2}\right)$ & 139 & 139 & 139 & 139 & 139 & 140 & 139 & 139 \\
\hline$r\left(\mathrm{C}_{2}-\mathrm{C}_{3}\right)$ & 141 & 140 & 140 & 141 & 140 & 140 & 142 & 141 \\
\hline$r\left(\mathrm{C}_{3}-\mathrm{C}_{4}\right)$ & 139 & 142 & 142 & 141 & 141 & 142 & 140 & 139 \\
\hline$r\left(\mathrm{C}_{4}-\mathrm{C}_{4 a}\right)$ & 140 & 139 & 139 & 139 & 139 & 139 & 139 & 140 \\
\hline$r\left(\mathrm{C}_{4 a}-\mathrm{C}_{9 a}\right)$ & 141 & 142 & 142 & 142 & 142 & 142 & 144 & 141 \\
\hline$r\left(\mathrm{C}_{10 a}-\mathrm{C}_{5}\right)$ & 140 & 139 & 139 & 139 & 139 & 139 & 140 & 140 \\
\hline$r\left(\mathrm{C}_{5}-\mathrm{C}_{6}\right)$ & 139 & 140 & 140 & 140 & 140 & 141 & 140 & 139 \\
\hline$r\left(\mathrm{C}_{6}-\mathrm{C}_{7}\right)$ & 141 & 140 & 140 & 140 & 140 & 140 & 141 & 141 \\
\hline$r\left(\mathrm{C}_{7}-\mathrm{C}_{8}\right)$ & 139 & 140 & 140 & 139 & 139 & 140 & 139 & 139 \\
\hline$r\left(\mathrm{C}_{8}-\mathrm{C}_{8 a}\right)$ & 141 & 141 & 141 & 142 & 142 & 141 & 141 & 141 \\
\hline$r\left(\mathrm{C}_{8 a}-\mathrm{C}_{10 a}\right)$ & 141 & 141 & 141 & 142 & 142 & 141 & 143 & 141 \\
\hline$r\left(\mathrm{C}_{8 a}-\mathrm{C}_{9}\right)$ & 148 & 147 & 147 & 143 & 143 & 147 & 144 & 148 \\
\hline$r\left(\mathrm{C}_{9}-\mathrm{C}_{9 a}\right)$ & 148 & 145 & 145 & 143 & 143 & 146 & 143 & 148 \\
\hline$r\left(\mathrm{C}_{9 a}-\mathrm{C}_{1}\right)$ & 141 & 143 & 143 & 142 & 142 & 142 & 144 & 141 \\
\hline$r\left(\mathrm{C}_{9}-\mathrm{O}\right)$ & 122 & 125 & 125 & 131 & 131 & 125 & 130 & 123 \\
\hline$r\left(\mathrm{C}_{4 a}-\mathrm{O}_{10}\right)$ & 136 & 136 & 136 & 136 & 136 & 137 & 136 & 136 \\
\hline$r\left(\mathrm{C}_{10 a}-\mathrm{O}_{10}\right)$ & 136 & 138 & 138 & 136 & 137 & 138 & 136 & 136 \\
\hline$r\left(\mathrm{C}_{3}-\mathrm{N}_{10^{\prime}}\right)$ & 143 & 146 & 146 & 143 & 143 & 145 & 143 & 143 \\
\hline$r\left(\mathrm{C}_{4 a^{\prime}}-\mathrm{N}_{10^{\prime}}\right)$ & 141 & 139 & 139 & 141 & 141 & 139 & 141 & 141 \\
\hline$r\left(\mathrm{C}_{1^{\prime}}-\mathrm{C}_{2^{\prime}}\right)$ & 140 & 140 & 140 & 140 & 140 & 138 & 140 & 140 \\
\hline$r\left(\mathrm{C}_{2^{\prime}}-\mathrm{C}_{3^{\prime}}\right)$ & 140 & 140 & 140 & 140 & 140 & 141 & 140 & 140 \\
\hline$r\left(\mathrm{C}_{3^{\prime}}-\mathrm{C}_{4^{\prime}}\right)$ & 139 & 138 & 138 & 139 & 139 & 140 & 139 & 139 \\
\hline$r\left(\mathrm{C}_{4^{\prime}}-\mathrm{C}_{4 a^{\prime}}\right)$ & 141 & 142 & 142 & 141 & 141 & 140 & 141 & 141 \\
\hline$r\left(\mathrm{C}_{4 a^{\prime}}-\mathrm{C}_{9 a^{\prime}}\right)$ & 141 & 143 & 143 & 141 & 141 & 143 & 141 & 141 \\
\hline$r\left(\mathrm{C}_{9 a^{\prime}}-\mathrm{C}_{1^{\prime}}\right)$ & 140 & 140 & 140 & 140 & 140 & 142 & 140 & 141 \\
\hline$r\left(\mathrm{C}_{9 a^{\prime}}-\mathrm{C}_{9^{\prime}}\right)$ & 154 & 152 & 152 & 154 & 154 & 153 & 154 & 154 \\
\hline
\end{tabular}

${ }^{a}$ isolated molecule

${ }^{b}$ in acetonitrile solution 
Table S2: Vertical DFT/MRCI-R excitation energies $\Delta E_{\text {vert }}$, oscillator strengths $f(r)$, and dipole moments $\mu$ at the ground-state geometry.

\begin{tabular}{lclrrr}
\hline \hline State & Excitation & Electronic structure & $\Delta E_{\text {vert }}(\mathbf{e V})$ & $f(r)$ & $\mu$ (Debye) \\
\hline \hline $1^{1} A^{\prime}$ & & $(0.93) \mathrm{GS}$ & 0.00 & - & 2.58 \\
$1^{1} A^{\prime \prime}$ & $\mathrm{CT}$ & $(0.80) \pi_{H} \rightarrow \pi_{L}^{*}$ & 3.09 & $4 \times 10^{-5}$ & 22.05 \\
$2^{1} A^{\prime \prime}$ & $\mathrm{LEX}$ & $(0.76) n_{\mathrm{O}} \rightarrow \pi_{L}^{*}$ & 3.61 & $<10^{-5}$ & 1.93 \\
$1^{1} A^{\prime}$ & $\mathrm{LEX}$ & $(0.70) \pi_{H-1} \rightarrow \pi_{L}^{*}$ & 3.83 & 0.13442 & 3.78 \\
\hline $1^{3} A^{\prime \prime}$ & $\mathrm{CT}$ & $(0.79) \pi_{H} \rightarrow \pi_{L}^{*}$ & 3.03 & - & 21.71 \\
$1^{3} A^{\prime}$ & $\mathrm{LEX}$ & $(0.63) \pi_{H-1} \rightarrow \pi_{L}^{*},(0.10) \pi_{H-6} \rightarrow \pi_{L}^{*}$ & 3.25 & - & 3.28 \\
$2^{3} A^{\prime \prime}$ & $\mathrm{LEA}$ & $(0.45) \pi_{H} \rightarrow \pi_{L+4}^{*},(0.18) \pi_{H} \rightarrow \pi_{L+5}^{*}$ & 3.35 & - & 2.54 \\
$3^{3} A^{\prime \prime}$ & $\mathrm{LEX}$ & $(0.76) n_{\mathrm{O}} \rightarrow \pi_{L}^{*}$ & 3.42 & - & 1.96 \\
& & 3.56 & - & 3.12 \\
$2^{3} A^{\prime}$ & $\mathrm{LEX}$ & $(0.46) \pi_{H-6} \rightarrow \pi_{L}^{*},(0.12) \pi_{H-1} \rightarrow \pi_{L}^{*}$ & 3.56 & - & 3.28 \\
\hline
\end{tabular}


Table S3: Vertical DFT/MRCI-R excitation energies $\Delta E_{\text {vert }}$, oscillator strengths $f(r)$, and dipole moments $\mu$ at the singlet CT geometry.

\begin{tabular}{lclrrr}
\hline \hline State & Excitation & Electronic structure & $\Delta E_{\text {vert }}(\mathbf{e V})$ & $f(r)$ & $\mu$ (Debye) \\
\hline \hline $1^{1} A^{\prime}$ & & $(0.92) \mathrm{GS}$ & 0.00 & - & 2.66 \\
$1^{1} A^{\prime \prime}$ & $\mathrm{CT}$ & $(0.81) \pi_{H} \rightarrow \pi_{L}^{*}$ & 2.77 & $5 \times 10^{-5}$ & 22.34 \\
$2^{1} A^{\prime \prime}$ & $\mathrm{LEX}$ & $(0.76) n_{\mathrm{O}} \rightarrow \pi_{L}^{*}$ & 3.35 & $<10^{-5}$ & 2.52 \\
$1^{1} A^{\prime}$ & $\mathrm{LEX}$ & $(0.68) \pi_{H-1} \rightarrow \pi_{L}^{*}$ & 3.69 & 0.13606 & 4.15 \\
\hline $1^{3} A^{\prime \prime}$ & $\mathrm{CT}$ & $(0.81) \pi_{H} \rightarrow \pi_{L}^{*}$ & 2.71 & - & 22.18 \\
$1^{3} A^{\prime}$ & $\mathrm{LEX}$ & $(0.48) \pi_{H-1} \rightarrow \pi_{L}^{*},(0.21) \pi_{H-6} \rightarrow \pi_{L}^{*}$ & 3.05 & - & 2.82 \\
$2^{3} A^{\prime \prime}$ & $\mathrm{LEX}$ & $(0.77) n_{\mathrm{O}} \rightarrow \pi_{L}^{*}$ & 3.17 & - & 2.58 \\
$3^{3} A^{\prime \prime}$ & $\mathrm{LEA}$ & $(0.52) \pi_{H} \rightarrow \pi_{L+3}^{*},(0.16) \pi_{H} \rightarrow \pi_{L+5}^{*}$ & 3.22 & - & 2.65 \\
\hline
\end{tabular}

Table S4: Vertical DFT/MRCI-R excitation energies $\Delta E_{\text {vert }}$, oscillator strengths $f(r)$, and dipole moments $\mu$ at the triplet CT geometry.

\begin{tabular}{lclrrr}
\hline \hline State & Excitation & Electronic structure & $\Delta E_{\text {vert }}(\mathrm{eV})$ & $f(r)$ & $\mu$ (Debye) \\
\hline \hline $1^{1} A^{\prime}$ & & $(0.92) \mathrm{GS}$ & 0.00 & - & 2.66 \\
$1^{1} A^{\prime \prime}$ & $\mathrm{CT}$ & $(0.81) \pi_{H} \rightarrow \pi_{L}^{*}$ & 2.77 & $1.4 \times 10^{-4}$ & 22.30 \\
$2^{1} A^{\prime \prime}$ & $\mathrm{LEX}$ & $(0.76) n_{\mathrm{O}} \rightarrow \pi_{L}^{*}$ & 3.35 & $<10^{-5}$ & 2.52 \\
$1^{1} A^{\prime}$ & $\mathrm{LEX}$ & $(0.68) \pi_{H-1} \rightarrow \pi_{L}^{*}$ & 3.69 & 0.13686 & 4.11 \\
\hline $1^{3} A^{\prime \prime}$ & $\mathrm{CT}$ & $(0.81) \pi_{H} \rightarrow \pi_{L}^{*}$ & 2.71 & - & 22.10 \\
$1^{3} A^{\prime}$ & $\mathrm{LEX}$ & $(0.48) \pi_{H-1} \rightarrow \pi_{L}^{*},(0.21) \pi_{H-6} \rightarrow \pi_{L}^{*}$ & 3.05 & - & 2.79 \\
$2^{3} A^{\prime \prime}$ & $\mathrm{LEX}$ & $(0.77) n_{\mathrm{O}} \rightarrow \pi_{L}^{*}$ & 3.17 & - & 2.58 \\
$3^{3} A^{\prime \prime}$ & $\mathrm{LEA}$ & $(0.52) \pi_{H} \rightarrow \pi_{L+3}^{*},(0.16) \pi_{H} \rightarrow \pi_{L+5}^{*}$ & 3.23 & - & 2.60 \\
\hline
\end{tabular}


Table S5: Vertical DFT/MRCI-R excitation energies $\Delta E_{\text {vert }}$, oscillator strengths $f(r)$, and dipole moments $\mu$ at the $\mathrm{S}\left(n_{\mathrm{O}} \pi_{L}^{*}\right)$ geometry.

\begin{tabular}{lclrrr}
\hline \hline State & Excitation & Electronic structure & $\Delta E_{\text {vert }}(\mathrm{eV})$ & $f(r)$ & $\mu$ (Debye) \\
\hline \hline $1^{1} A^{\prime}$ & & $(0.92) \mathrm{GS}$ & 0.00 & - & 2.93 \\
$1^{1} A^{\prime \prime}$ & LEX, CT & $(0.47) n_{\mathrm{O}} \rightarrow \pi_{L}^{*},(0.32) \pi_{H} \rightarrow \pi_{L}^{*}$ & 2.80 & $1 \times 10^{-5}$ & 7.65 \\
$2^{1} A^{\prime \prime}$ & $\mathrm{CT}, \mathrm{LEX}$ & $(0.49) \pi_{H} \rightarrow \pi_{L}^{*},(0.47) n_{\mathrm{O}} \rightarrow \pi_{L}^{*}$ & 2.85 & $2 \times 10^{-5}$ & 13.46 \\
$1^{1} A^{\prime}$ & $\mathrm{LEX}$ & $(0.71) \pi_{H-1} \rightarrow \pi_{L}^{*}$ & 3.59 & 0.16079 & 4.05 \\
\hline $1^{3} A^{\prime}$ & $\mathrm{LEX}$ & $(0.41) \pi_{H-1} \rightarrow \pi_{L}^{*},(0.27) \pi_{H-5} \rightarrow \pi_{L}^{*}$ & 2.64 & - & 1.78 \\
$1^{3} A^{\prime \prime}$ & $\mathrm{LEX}$ & $(0.73) n_{\mathrm{O}} \rightarrow \pi_{L}^{*}$ & 2.67 & - & 0.78 \\
$2^{3} A^{\prime \prime}$ & $\mathrm{CT}$ & $(0.76) \pi_{H} \rightarrow \pi_{L+1}^{*}$ & 2.78 & - & 21.56 \\
$1^{3} A^{\prime}$ & $\mathrm{LEX}$ & $(0.35) \pi_{H-5} \rightarrow \pi_{L}^{*},(0.35) \pi_{H-1} \rightarrow \pi_{L}^{*}$ & 3.28 & - & 4.69 \\
$3^{3} A^{\prime}$ & $\mathrm{LEA}$ & $(0.49) \pi_{H} \rightarrow \pi_{L+4}^{*},(0.14) \pi_{H} \rightarrow \pi_{L+5}^{*}$ & 3.38 & - & 2.85 \\
\hline
\end{tabular}

Table S6: Vertical DFT/MRCI-R excitation energies $\Delta E_{\text {vert }}$, oscillator strengths $f(r)$, and dipole moments $\mu$ at the $\mathrm{T}\left(n_{\mathrm{O}} \pi_{L}^{*}\right)$ geometry.

\begin{tabular}{lclrrr}
\hline \hline State & Excitation & Electronic structure & $\Delta E_{\text {vert }}(\mathrm{eV})$ & $f(r)$ & $\mu$ (Debye) \\
\hline \hline $1^{1} A^{\prime}$ & & $(0.92) \mathrm{GS}$ & 0.00 & - & 2.94 \\
$1^{1} A^{\prime \prime}$ & LEX, CT & $(0.66) n_{\mathrm{O}} \rightarrow \pi_{L}^{*},(0.12) \pi_{H} \rightarrow \pi_{L}^{*}$ & 2.77 & $1 \times 10^{-5}$ & 1.66 \\
$2^{1} A^{\prime \prime}$ & $\mathrm{CT}, \mathrm{LEX}$ & $(0.69) \pi_{H} \rightarrow \pi_{L}^{*},(0.12) n_{\mathrm{O}} \rightarrow \pi_{L}^{*}$ & 2.83 & $3 \times 10^{-5}$ & 19.67 \\
$1^{1} A^{\prime}$ & $\mathrm{LEX}$ & $(0.71) \pi_{H-1} \rightarrow \pi_{L}^{*}$ & 3.58 & 0.16206 & 4.08 \\
\hline $1^{3} A^{\prime}$ & $\mathrm{LEX}$ & $(0.41) \pi_{H-1} \rightarrow \pi_{L}^{*},(0.28) \pi_{H-5} \rightarrow \pi_{L}^{*}$ & 2.61 & - & 1.90 \\
$1^{3} A^{\prime \prime}$ & $\mathrm{LEX}$ & $(0.75) n_{\mathrm{O}} \rightarrow \pi_{L}^{*}$ & 2.63 & - & 1.68 \\
$2^{3} A^{\prime \prime}$ & $\mathrm{CT}$ & $(0.78) \pi_{H} \rightarrow \pi_{L+1}^{*}$ & 2.77 & - & 22.47 \\
$1^{3} A^{\prime}$ & $\mathrm{LEX}$ & $(0.36) \pi_{H-5} \rightarrow \pi_{L}^{*},(0.35) \pi_{H-1} \rightarrow \pi_{L}^{*}$ & 3.28 & - & 4.72 \\
$3^{3} A^{\prime}$ & $\mathrm{LEA}$ & $(0.48) \pi_{H} \rightarrow \pi_{L+4}^{*},(0.15) \pi_{H} \rightarrow \pi_{L+5}^{*}$ & 3.38 & - & 2.83 \\
\hline
\end{tabular}


Table S7: Vertical DFT/MRCI-R excitation energies $\Delta E_{\text {vert }}$, oscillator strengths $f(r)$, and dipole moments $\mu$ at the $\mathrm{S}\left(\pi_{H-1} \pi_{L}^{*}\right)$ geometry.

\begin{tabular}{lclrrr}
\hline \hline State & Excitation & Electronic structure & $\Delta E_{\text {vert }}(\mathbf{e V})$ & $f(r)$ & $\mu$ (Debye) \\
\hline \hline $1^{1} A^{\prime}$ & & $(0.92) \mathrm{GS}$ & 0.00 & - & 2.66 \\
$1^{1} A^{\prime \prime}$ & $\mathrm{CT}$ & $(0.81) \pi_{H} \rightarrow \pi_{L}^{*}$ & 2.87 & $5 \times 10^{-5}$ & 22.54 \\
$2^{1} A^{\prime \prime}$ & $\mathrm{LEX}$ & $(0.76) n_{\mathrm{O}} \rightarrow \pi_{L}^{*}$ & 3.34 & $<10^{-5}$ & 2.38 \\
$1^{1} A^{\prime}$ & $\mathrm{LEX}$ & $(0.21) \pi_{H-1} \rightarrow \pi_{L}^{*},(0.21) \pi_{H-3} \rightarrow \pi_{L}^{*}$ & 3.60 & 0.13645 & 4.22 \\
\hline $1^{3} A^{\prime \prime}$ & $\mathrm{CT}$ & $(0.80) \pi_{H} \rightarrow \pi_{L}^{*}$ & 2.81 & - & 22.34 \\
$1^{3} A^{\prime}$ & $\mathrm{LEX}$ & $(0.44) \pi_{H-1} \rightarrow \pi_{L}^{*},(0.18) \pi_{H-3} \rightarrow \pi_{L}^{*}$, & 3.00 & - & 3.37 \\
& & $(0.15) \pi_{H-6} \rightarrow \pi_{L}^{*}$ & & \\
$2^{3} A^{\prime \prime}$ & $\mathrm{LEX}$ & $(0.77) n_{\mathrm{O}} \rightarrow \pi_{L}^{*}$ & 3.17 & - & 2.43 \\
$3^{3} A^{\prime \prime}$ & $\mathrm{LEA}$ & $(0.41) \pi_{H} \rightarrow \pi_{L+4}^{*},(0.15) \pi_{H} \rightarrow \pi_{L+5}^{*}$, & 3.26 & - & 2.65 \\
& & $(0.13) \pi_{H} \rightarrow \pi_{L+2}^{*}$ & & & - \\
\hline
\end{tabular}

Table S8: Vertical DFT/MRCI-R excitation energies $\Delta E_{\text {vert }}$, oscillator strengths $f(r)$, and dipole moments $\mu$ at the $\mathrm{T}\left(\pi_{H-1} \pi_{L}^{*}\right)$ geometry.

\begin{tabular}{lclrrr}
\hline \hline State & Excitation & Electronic structure & $\Delta E_{\text {vert }}(\mathrm{eV})$ & $f(r)$ & $\mu$ (Debye) \\
\hline \hline $1^{1} A^{\prime}$ & & $(0.92) \mathrm{GS}$ & 0.00 & - & 3.06 \\
$1^{1} A^{\prime \prime}$ & $\mathrm{CT}$ & $(0.76) \pi_{H} \rightarrow \pi_{L}^{*}$ & 2.84 & $3 \times 10^{-5}$ & 21.58 \\
$2^{1} A^{\prime \prime}$ & $\mathrm{LEX}$ & $(0.72) n_{\mathrm{O}} \rightarrow \pi_{L}^{*}$ & 2.94 & $<10^{-5}$ & 0.53 \\
$1^{1} A^{\prime}$ & $\mathrm{LEX}$ & $(0.73) \pi_{H-1} \rightarrow \pi_{L}^{*}$ & 3.55 & 0.16385 & 4.16 \\
\hline $1^{3} A^{\prime}$ & $\mathrm{LEX}$ & $(0.51) \pi_{H-1} \rightarrow \pi_{L}^{*},(0.22) \pi_{H-5} \rightarrow \pi_{L}^{*}$ & 2.67 & - & 2.10 \\
$1^{3} A^{\prime \prime}$ & $\mathrm{CT}, \mathrm{LEX}$ & $(0.42) \pi_{H} \rightarrow \pi_{L+1}^{*},(0.37) n_{\mathrm{O}} \rightarrow \pi_{L}^{*}$ & 2.77 & - & 10.85 \\
$2^{3} A^{\prime \prime}$ & LEX, CT & $(0.40) n_{\mathrm{O}} \rightarrow \pi_{L}^{*},(0.38) \pi_{H} \rightarrow \pi_{L+1}^{*}$ & 2.82 & - & 10.16 \\
$1^{3} A^{\prime}$ & $\mathrm{LEX}$ & $(0.44) \pi_{H-5} \rightarrow \pi_{L}^{*},(0.27) \pi_{H-1} \rightarrow \pi_{L}^{*}$ & 3.34 & - & 4.47 \\
$3^{3} A^{\prime}$ & LEA & $(0.49) \pi_{H} \rightarrow \pi_{L+4}^{*},(0.13) \pi_{H} \rightarrow \pi_{L+5}^{*}$ & 3.39 & - & 2.98 \\
\hline
\end{tabular}


Table S9: Selected energy differences $\Delta E(\mathrm{~S}-\mathrm{T})$ and spin-orbit matrix elements (SOMEs) $\left[\mathrm{cm}^{-1}\right]$ of ACRXTN at the relaxed ${ }^{1}\left(\pi_{H} \rightarrow \pi_{L}^{*}\right)$ (singlet CT) TDDFT geometry in vacuum using DFT/MRCI-R wave functions.

\begin{tabular}{|c|c|c|}
\hline$\Delta E(\mathbf{S}-\mathbf{T})\left(\mathbf{c m}^{-1}\right)$ & & $\operatorname{SOME}\left(\mathrm{cm}^{-1}\right)$ \\
\hline \multirow[t]{3}{*}{416} & $\left|\left\langle\mathrm{~S}_{n \pi^{*}}\left|\hat{\mathcal{H}}_{\mathrm{SOx}}\right| \mathrm{T}_{n \pi^{*}}\right\rangle\right|$ & $0.013 i$ \\
\hline & $\left|\left\langle\mathrm{S}_{n \pi^{*}}\left|\hat{\mathcal{H}}_{\mathrm{SOy}}\right| \mathrm{T}_{n \pi^{*}}\right\rangle\right|$ & $-0.021 i$ \\
\hline & $\left|\left\langle\mathrm{S}_{n \pi^{*}}\left|\hat{\mathcal{H}}_{\mathrm{SOz}}\right| \mathrm{T}_{n \pi^{*}}\right\rangle\right|$ & $-0.006 i$ \\
\hline \multirow{3}{*}{-3217} & $\left|\left\langle\mathrm{~S}_{C T}\left|\hat{\mathcal{H}}_{\mathrm{SOx}}\right| \mathrm{T}_{n \pi^{*}}\right\rangle\right|$ & $0.042 i$ \\
\hline & $\left|\left\langle\mathrm{S}_{C T}\left|\hat{\mathcal{H}}_{\mathrm{SOy}}\right| \mathrm{T}_{n \pi^{*}}\right\rangle\right|$ & $-0.058 i$ \\
\hline & $\left|\left\langle\mathrm{S}_{C T}\left|\hat{\mathcal{H}}_{\mathrm{SOz}}\right| \mathrm{T}_{n \pi^{*}}\right\rangle\right|$ & $-0.245 i$ \\
\hline \multirow[t]{3}{*}{2369} & $\left|\left\langle\mathrm{~S}_{n \pi^{*}}\left|\hat{\mathcal{H}}_{\mathrm{SOx}}\right| \mathrm{T}_{\pi \pi^{*}}\right\rangle\right|$ & $-14.165 i$ \\
\hline & $\left|\left\langle\mathrm{S}_{n \pi^{*}}\left|\hat{\mathcal{H}}_{\mathrm{SOy}}\right| \mathrm{T}_{\pi \pi^{*}}\right\rangle\right|$ & $29.744 i$ \\
\hline & $\left|\left\langle\mathrm{S}_{n \pi^{*}}\left|\hat{\mathcal{H}}_{\mathrm{SOx}}\right| \mathrm{T}_{\pi \pi^{*}}\right\rangle\right|$ & $7.7221 i$ \\
\hline \multirow[t]{3}{*}{-2264} & $\left|\left\langle\mathrm{~S}_{C T}\left|\hat{\mathcal{H}}_{\mathrm{SOx}}\right| \mathrm{T}_{\pi \pi^{*}}\right\rangle\right|$ & $0.281 i$ \\
\hline & $\left|\left\langle\mathrm{S}_{C T}\left|\hat{\mathcal{H}}_{\mathrm{SOy}}\right| \mathrm{T}_{\pi \pi^{*}}\right\rangle\right|$ & $-1.239 i$ \\
\hline & $\left|\left\langle\mathrm{S}_{C T}\left|\hat{\mathcal{H}}_{\mathrm{SOz}}\right| \mathrm{T}_{\pi \pi^{*}}\right\rangle\right|$ & $0.269 i$ \\
\hline \multirow[t]{3}{*}{5112} & $\left|\left\langle\mathrm{~S}_{n \pi^{*}}\left|\hat{\mathcal{H}}_{\mathrm{SOx}}\right| \mathrm{T}_{C T}\right\rangle\right|$ & $-0.063 i$ \\
\hline & $\left|\left\langle\mathrm{S}_{n \pi^{*}}\left|\hat{\mathcal{H}}_{\mathrm{SOy}}\right| \mathrm{T}_{C T}\right\rangle\right|$ & $0.101 i$ \\
\hline & $\left|\left\langle\mathrm{S}_{n \pi^{*}}\left|\hat{\mathcal{H}}_{\mathrm{SOz}}\right| \mathrm{T}_{C T}\right\rangle\right|$ & $0.215 i$ \\
\hline \multirow[t]{3}{*}{479} & $\left|\left\langle\mathrm{~S}_{C T}\left|\hat{\mathcal{H}}_{\mathrm{SOx}}\right| \mathrm{T}_{C T}\right\rangle\right|$ & $-0.005 i$ \\
\hline & $\left|\left\langle\mathrm{S}_{C T}\left|\hat{\mathcal{H}}_{\mathrm{SOy}}\right| \mathrm{T}_{C T}\right\rangle\right|$ & $0.008 i$ \\
\hline & $\left|\left\langle\mathrm{S}_{C T}\left|\hat{\mathcal{H}}_{\mathrm{SOz}}\right| \mathrm{T}_{C T}\right\rangle\right|$ & $0.035 i$ \\
\hline
\end{tabular}


Table S10: Selected energy differences $\Delta E(\mathrm{~S}-\mathrm{T})$ and spin-orbit matrix elements (SOMEs) $\left[\mathrm{cm}^{-1}\right]$ of ACRXTN at the relaxed ${ }^{1}\left(n_{\mathrm{O}} \pi_{L}^{*}\right)$ TDDFT geometry in vacuum using DFT/MRCI$\mathrm{R}$ wave functions.

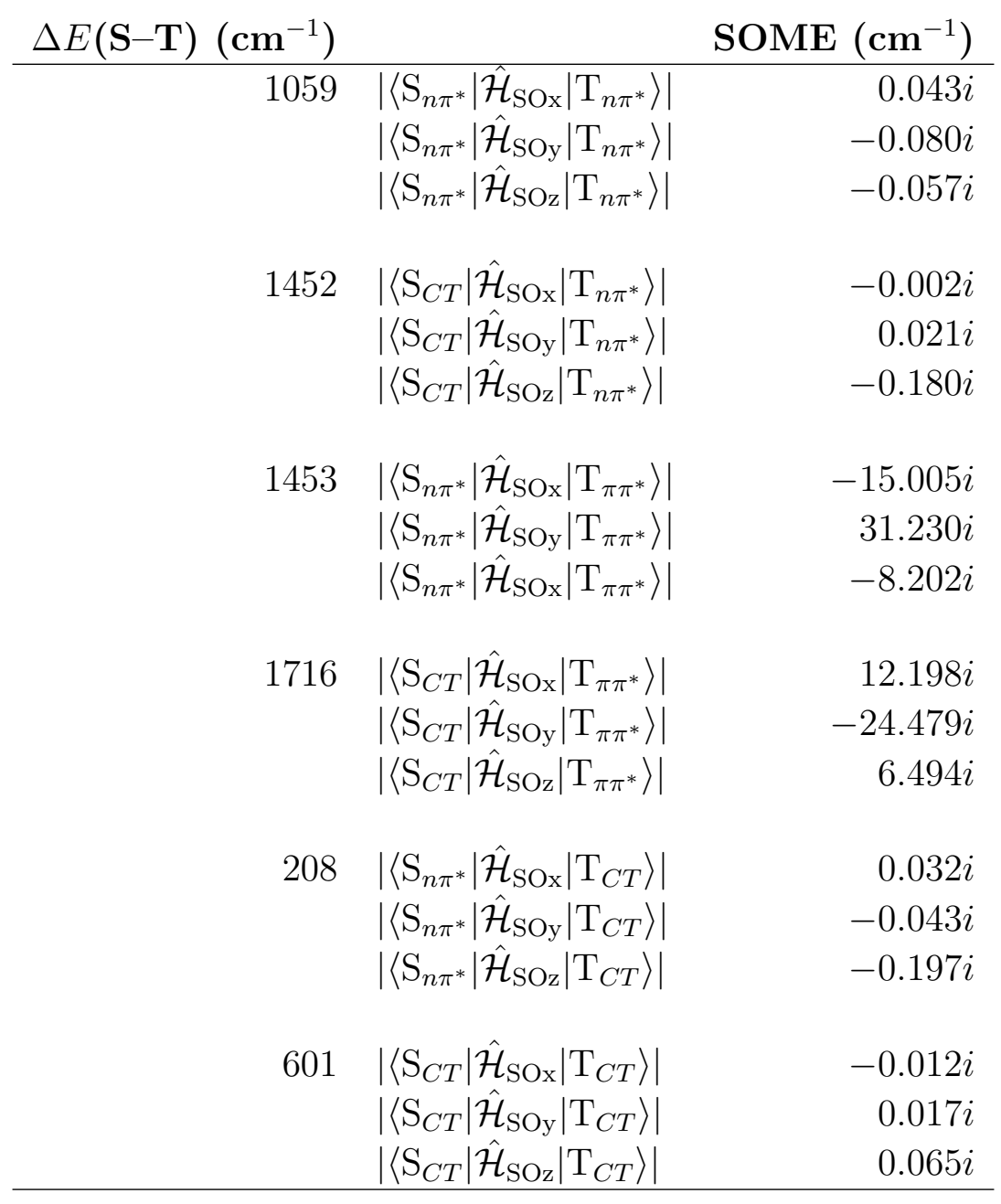


Table S11: Selected energy differences $\Delta E(\mathrm{~S}-\mathrm{T})$ and spin-orbit matrix elements (SOMEs) $\left[\mathrm{cm}^{-1}\right]$ of ACRXTN at the relaxed ${ }^{3}\left(\pi_{H-1} \pi_{L}^{*}\right)$ UDFT geometry in vacuum using DFT/MRCI$\mathrm{R}$ wave functions.

\begin{tabular}{|c|c|c|}
\hline$\Delta E(\mathbf{S}-\mathbf{T})\left(\mathbf{c m}^{-1}\right)$ & & $\operatorname{SOME}\left(\mathrm{cm}^{-1}\right)$ \\
\hline \multirow[t]{3}{*}{1028} & $\left|\left\langle\mathrm{~S}_{n \pi^{*}}\left|\hat{\mathcal{H}}_{\mathrm{SOx}}\right| \mathrm{T}_{n \pi^{*}}\right\rangle\right|$ & 0.000 \\
\hline & $\left|\left\langle\mathrm{S}_{n \pi^{*}}\left|\hat{\mathcal{H}}_{\mathrm{SOy}}\right| \mathrm{T}_{n \pi^{*}}\right\rangle\right|$ & 0.000 \\
\hline & $\left|\left\langle\mathrm{S}_{n \pi^{*}}\left|\hat{\mathcal{H}}_{\mathrm{SOz}}\right| \mathrm{T}_{n \pi^{*}}\right\rangle\right|$ & -0.111 \\
\hline \multirow[t]{3}{*}{200} & $\left|\left\langle\mathrm{~S}_{C T}\left|\hat{\mathcal{H}}_{\mathrm{SOx}}\right| \mathrm{T}_{n \pi^{*}}\right\rangle\right|$ & $0.000 i$ \\
\hline & $\left|\left\langle\mathrm{S}_{C T}\left|\hat{\mathcal{H}}_{\mathrm{SOy}}\right| \mathrm{T}_{n \pi^{*}}\right\rangle\right|$ & $0.000 i$ \\
\hline & $\left|\left\langle\mathrm{S}_{C T}\left|\hat{\mathcal{H}}_{\mathrm{SOz}}\right| \mathrm{T}_{n \pi^{*}}\right\rangle\right|$ & $0.208 i$ \\
\hline \multirow[t]{3}{*}{2175} & $\left|\left\langle\mathrm{~S}_{n \pi^{*}}\left|\hat{\mathcal{H}}_{\mathrm{SOx}}\right| \mathrm{T}_{\pi \pi^{*}}\right\rangle\right|$ & $-17.874 i$ \\
\hline & $\left|\left\langle\mathrm{S}_{n \pi^{*}}\left|\hat{\mathcal{H}}_{\mathrm{SOy}}\right| \mathrm{T}_{\pi \pi^{*}}\right\rangle\right|$ & $37.366 i$ \\
\hline & $\left|\left\langle\mathrm{S}_{n \pi^{*}}\left|\hat{\mathcal{H}}_{\mathrm{SOx}}\right| \mathrm{T}_{\pi \pi^{*}}\right\rangle\right|$ & $0.000 i$ \\
\hline \multirow[t]{3}{*}{1347} & $\left|\left\langle\mathrm{~S}_{C T}\left|\hat{\mathcal{H}}_{\mathrm{SOx}}\right| \mathrm{T}_{\pi \pi^{*}}\right\rangle\right|$ & $-4.288 i$ \\
\hline & $\left|\left\langle\mathrm{S}_{C T}\left|\hat{\mathcal{H}}_{\mathrm{SOy}}\right| \mathrm{T}_{\pi \pi^{*}}\right\rangle\right|$ & $9.634 i$ \\
\hline & $\left|\left\langle\mathrm{S}_{C T}\left|\hat{\mathcal{H}}_{\mathrm{SOz}}\right| \mathrm{T}_{\pi \pi^{*}}\right\rangle\right|$ & $0.000 i$ \\
\hline \multirow[t]{3}{*}{1432} & $\left|\left\langle\mathrm{~S}_{n \pi^{*}}\left|\hat{\mathcal{H}}_{\mathrm{SOx}}\right| \mathrm{T}_{C T}\right\rangle\right|$ & $0.000 i$ \\
\hline & $\left|\left\langle\mathrm{S}_{n \pi^{*}}\left|\hat{\mathcal{H}}_{\mathrm{SOy}}\right| \mathrm{T}_{C T}\right\rangle\right|$ & $0.000 i$ \\
\hline & $\left|\left\langle\mathrm{S}_{n \pi^{*}}\left|\hat{\mathcal{H}}_{\mathrm{SOz}}\right| \mathrm{T}_{C T}\right\rangle\right|$ & $0.186 i$ \\
\hline \multirow[t]{3}{*}{604} & $\left|\left\langle\mathrm{~S}_{C T}\left|\hat{\mathcal{H}}_{\mathrm{SOx}}\right| \mathrm{T}_{C T}\right\rangle\right|$ & $0.000 i$ \\
\hline & $\left|\left\langle\mathrm{S}_{C T}\left|\hat{\mathcal{H}}_{\mathrm{SOy}}\right| \mathrm{T}_{C T}\right\rangle\right|$ & $0.000 i$ \\
\hline & $\left|\left\langle\mathrm{S}_{C T}\left|\hat{\mathcal{H}}_{\mathrm{SOz}}\right| \mathrm{T}_{C T}\right\rangle\right|$ & $-0.085 i$ \\
\hline
\end{tabular}

